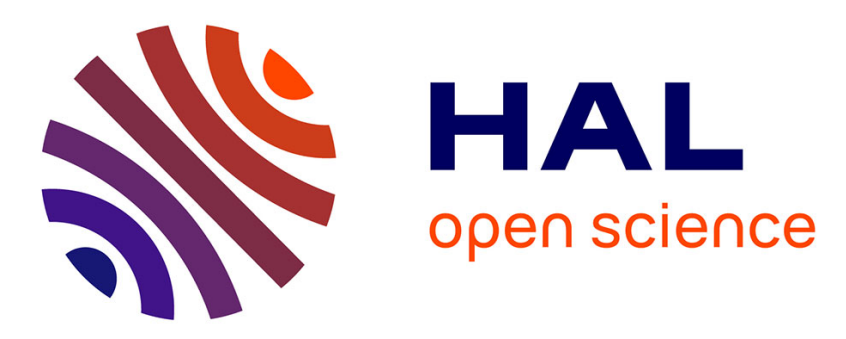

\title{
Bubble Solution Description by Non-Extensive Thermodynamics: Pressure Effect
}

Pierre Letellier, Mireille Turmine

\section{To cite this version:}

Pierre Letellier, Mireille Turmine. Bubble Solution Description by Non-Extensive Thermodynamics: Pressure Effect. ChemPhysChem, 2019, 20 (17), pp.2230-2235. 10.1002/cphc.201900412 . hal02281737

\section{HAL Id: hal-02281737 \\ https: / hal.sorbonne-universite.fr/hal-02281737}

Submitted on 9 Sep 2019

HAL is a multi-disciplinary open access archive for the deposit and dissemination of scientific research documents, whether they are published or not. The documents may come from teaching and research institutions in France or abroad, or from public or private research centers.
L'archive ouverte pluridisciplinaire HAL, est destinée au dépôt et à la diffusion de documents scientifiques de niveau recherche, publiés ou non, émanant des établissements d'enseignement et de recherche français ou étrangers, des laboratoires publics ou privés. 


\title{
Bubble solution description by nonextensive Thermodynamics. Pressure effect
}

\author{
Pierre Letellier ${ }^{[\mathrm{a}]}$, Mireille Turmine* ${ }^{* \mathrm{a}]}$
}

\begin{abstract}
We showed in this study that nanobubble solutions should not be considered as the simple juxtaposition of autonomous phases (a solution and bubbles) but as particular entities, i.e., "supersaturated solutions" where gas is simultaneously in two forms in permanent exchange. Gibbs' extensive Thermodynamics cannot claim to describe legitimately their behavior.

In this work, we showed how the use of the nonextensive thermodynamics allows describing the physicochemical properties of such media, some of which are counter-intuitive. Thus, an increase in pressure can result in an increase in the bubble size, contrary to what is provided by Boyle-Mariotte's law. The theoretical relationships proposed in this work constitute another approach to bubble solutions, which considers the non-autonomous nature of the components of supersaturated gas solutions and their "nonextensive" nature.
\end{abstract}

[a] Prof. P. Letellier, Dr M. Turmine Sorbonne Université, CNRS, Laboratoire Interfaces et Systèmes Électrochimiques (LISE),

4, place Jussieu, 75005 Paris, France

mireille.turmine@sorbonne-universite.fr 


\section{Introduction}

The large number of publications, sometimes contradictory, concerning the behavior of micro and nanobubble solutions in water and other solvents ${ }^{[1]}$, shows that the subject is still scientifically immature $^{[2]}$. The stability of these media, their longevity, reactivity, structure continue to intrigue many researchers ${ }^{[3]}$ who generally approach the physicochemical study of these media from Gibbs' extensive thermodynamics.

For our part, we are reserved on this approach, which does not consider the fact that the interfacial energies involved in these systems are necessarily nonextensive. This was explained in an earlier study on the solubility of "confined gases" ${ }^{[4]}$, conducted using the "nonextensive Thermodynamics", (NET) ${ }^{[5]}$, the basis of which was laid in 2004. We have shown that, in these systems, gas is in a partition equilibrium between the bubbles and the medium (or media) in contact, and that as such we must wonder on the autonomy of the phases present, in the sense of Defay ${ }^{[6]}$. It is recalled that in order to have an autonomous phase, the variables that define the system must be independent, which requires that their values can be freely set. However, particle size is not a controllable parameter. It is a consequence of the other variables of the environment (external pressure, composition, temperature, nature of the constituents, ...).

It therefore appears that, in the general case, the usual laws of extensive thermodynamics, used to describe the autonomous phases, are not suitable to describe the behavior of these bubble solutions.

To strengthen our analysis on this point, we propose, in this work, a complementary thought on the pressure and dilution effects applied to these media. If a bubble solution is considered as a simple juxtaposition of two autonomous systems (gas and solvent), and we wonder on the effect of the surrounding pressure on the system, the Boyle Mariotte's law is logically used which predicts a decrease in the bubble volume, when the pressure increases. If we approach this same problem, through the non-extensive Thermodynamics, by assuming that the phases are not autonomous, we can predict an evolution in the opposite direction, i.e., an increase in the bubbles' size with the pressure.

It seemed important to us to discuss in detail this counter-intuitive evolution of the system and to deduce from it some knowledge about the behavior of these systems. 


\section{The nonextensive Thermodynamics applied to confined gases}

\subsection{Pressure in a nonextensive gaseous phase}

Let suppose a solution in which the gas nanobubbles are included. In the context of nonextensive Thermodynamics, no attempt is made to specify the geometric outlines of these gaseous entities. They are considered as particles, of volume $\mathrm{V}$, whose interface with the surrounding medium is not geometrically defined ("fuzzy interface"). Bubbles are a nonextensive phase, (Figure 1). The main theoretical advance we have made for the description and understanding of the behaviour of such systems, in 2004, has been to demonstrate that the pressure in a nonextensive phase, $P_{N E}$, is different from the external pressure, $P$, when an energy, $E_{\sigma}$, is associated to the interface and defined as the product of two terms:

$$
d E_{\sigma}=\tau d \chi
$$

$\chi$ is a characteristic "extensity" of the fuzzy interface. In the general case, the value of $\chi$ does not vary proportionally with the considered system mass. It is therefore not extensive.

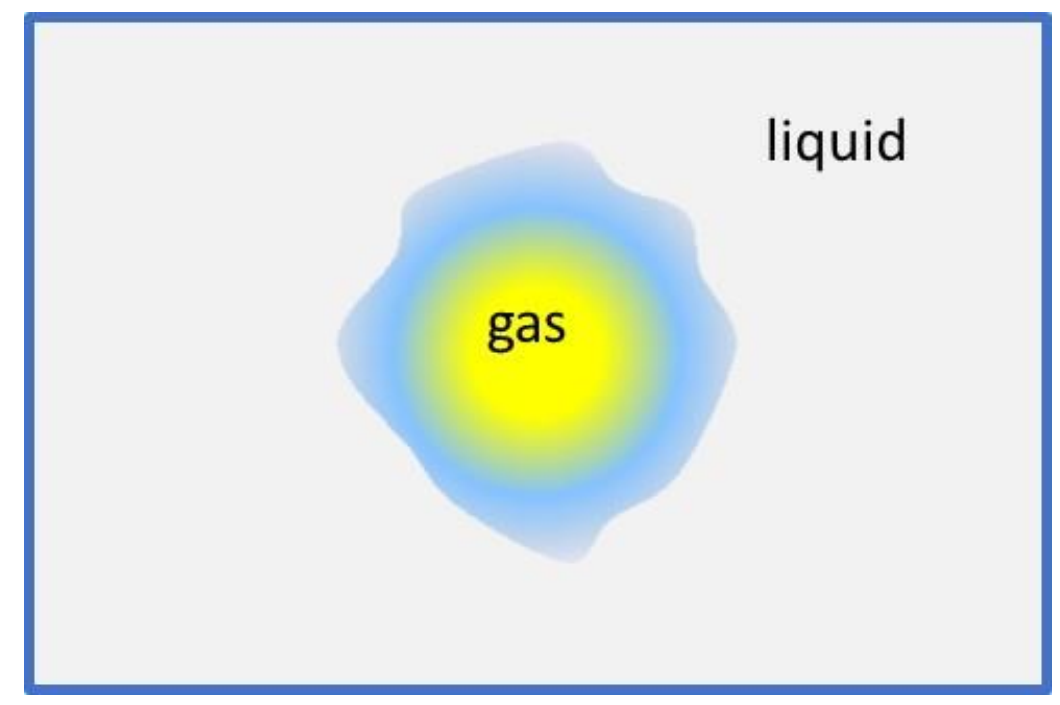

Figure 1: The gas bubble is the nonextensive phase in contact with the medium through an ill-defined interface (fuzzy interface).

Conventionally, $\tau$ is an intensive variable associated with the extensivity $\chi$. Its value can be positive, null or negative ${ }^{[7]}$. It does not depend on the system mass. In such conditions, $E_{\sigma}$ is not an extensive magnitude. In the general case, it is shown that, for a system involving a single interface with its environment, the pressure difference between the nonextensive phase $P_{N E}$, which cannot be controlled, and the external phase, $P$, which can be controlled, is written

$$
P_{N E}-P=\tau \frac{d \chi}{d V}
$$

$V$ is the volume of the nonextensive phase. 
For condensed phases, and for the gases at constant pressure, $V$ is an extensive magnitude. Its value varies proportionally with the system mass. By convention, we have assumed that $\chi$ is an Euler function of degree $\alpha(0 \leq \alpha \leq 1)$ of the system mass and therefore of its volume.

$$
\begin{aligned}
& \chi=\chi(V) \\
& \chi_{\lambda}=\chi(\lambda V)=\lambda^{\alpha} \chi(V)=\lambda^{\alpha} \chi
\end{aligned}
$$

$\lambda$ is any number. The parameter $\alpha$ being positive, the value of $\chi$ increases as the system volume increases. One of the simplest ways to mathematically account for this property is to lay down that $\chi$ varies with $V$ according to a power law.

We therefore conventionally pose

$$
\chi=a V^{\alpha}
$$

$a$ is a parameter that takes into account the nature and spatial characteristics of the particle. So, in such conditions, since the volume $V$ is expressed in $\mathrm{m}^{3}$, the extent $\chi$ is in $\mathrm{m}^{3 \alpha}$. The following relationship can be deduced for the derivatives

$$
\frac{d \chi}{d V}=\alpha \frac{\chi}{V}=a \alpha V^{\alpha-1}
$$

The expression of the pressure difference between the nonextensive phase and the external pressure can be thus established

$$
P_{N E}-P=\tau \frac{d \chi}{d V}=a \alpha \tau V^{\alpha-1}=\Omega V^{\alpha-1}
$$

$\Omega$ is a specific constant of the considered system whose unit is expressed in $\mathrm{J} \mathrm{m}^{-3 \alpha}$. It can be positive or negative. The pressure difference between the nonextensive phase and the environment is directly related to the particle volume, its unit is in $\mathrm{Pa}$.

As we have shown in our previous work, this relationship simply allows finding the expression of Laplace's law for a spherical system with a radius $r$. In this situation, the extensity $\chi$ is identified with the sphere area and $\tau$ with the surface tension. The area is then a homogeneous function of degree $\alpha=2 / 3$ of the volume and the pressure difference between the nonextensive phase and the external pressure varies as the inverse of the radius.

$$
P_{N E}-P=\gamma \frac{d A}{d V}=\gamma \frac{d\left(4 \pi r^{2}\right)}{d\left(\frac{4}{3} \pi r^{3}\right)}=\frac{2 \gamma}{r}=\frac{2 \gamma}{\left(\frac{3}{4 \pi}\right)^{1 / 3}} V^{-1 / 3}=\Omega_{\text {sphere }} V^{-1 / 3}
$$

In this particular case, since the interfacial tension is generally positive ${ }^{[8]}$, the internal pressure of the spherical particle is higher than the external pressure. This example shows that the radius is not an independent variable. 


\subsection{Behaviour of bubbles in supersaturated solutions}

When a liquid and a gas are brought into contact, the gas partially solubilizes in the liquid. Henry's law, whose relationship will be later clarified, characterizes this equilibrium when the phases are "unlimited". When considering bubbles, there is no reason for the phenomenon to be different. It is then shown that, if $\Omega$ is positive, the gas concentrations in solution at equilibrium are higher than Henry's concentration. In this case, the solutions are "oversaturated". We therefore assume that the exchange between the solubilized gas and that present in the bubbles is reached and that a thermodynamic equilibrium is achieved.

\subsubsection{Thermodynamic condition of solubility}

Knowing the expression of the pressure of the internal nonextensive phase of the particle, we reach that of its chemical potential. For a pure gas $i$, constituting a nonextensive phase at the pressure, $P_{N E}$, (with $P$ the external pressure), we write

$$
\mu_{i}=\mu_{i}^{o}+R T \ln \left\{f_{i}\right\}=\mu_{i}^{o}+R T \ln \left\{{ }^{g} \gamma_{i} P_{N E}\right\}=\mu_{i}^{o}+R T \ln \left(\left\{{ }^{g} \gamma_{i}\right\}\left\{P+\Omega_{i} V^{\alpha-1}\right\}\right)
$$

$\mu_{i}^{o}$ is the standard chemical potential of the gas, at standard pressure, $\left\{f_{i}\right\}$ is the value of the gas fugacity, $\left\{{ }^{g} \gamma_{i}\right\}$ is the value of the gas fugacity coefficient at considered temperature and pressure. $\Omega_{i}$ is a specific parameter for the gas $i$.

In our approach, we consider that the gas in the particle is in equilibrium of solubility with the gas in solution at the external pressure $P$. Its molar concentration is ${ }^{P} C_{i, s a t}$

$$
\mu_{i}={ }^{P} \mu_{i}^{\infty}+R T \ln \left\{{ }^{P} a_{i, s a t}\right\} \approx{ }^{P} \mu_{i}^{\infty}+R T \ln \left(\left\{{ }^{s o l} \gamma_{i}\right\}\left\{{ }^{P} C_{i, s a t}\right\}\right)
$$

${ }^{P} \mu_{i}^{\infty}$ is the standard chemical potential of the gas i solubilized at the external pressure, $P$. This extent varies with the pressure $P$, according to

$$
\left(\frac{\partial \mu_{i}^{\infty}}{\partial P}\right)_{T, n_{i}}=V_{i}^{\infty}
$$

$V_{i}^{\infty}$ is the partial molar volume of the gas at infinite dilution (in $\left.\mathrm{m}^{3} \mathrm{~mol}^{-1}\right) .\left\{{ }^{P} a_{i, s a t}\right\}$ is the value of the activity of $i$ at saturation at pressure $\mathrm{P}$ and $\left\{{ }^{s o l} \gamma_{i}\right\}$ is the activity coefficient of $i$ in solution, referred to the infinite diluted state in the molar concentration scale.

The equilibrium condition of the system is written, at the external pressure $P$, by assuming that its affinity is zero, i.e. by equalizing the chemical potentials for the gas solubilized in solution and that present into the bubbles.

$$
\mu_{i}=\mu_{i}^{o}+R T \ln \left(\left\{{ }^{g} \gamma_{i}\right\}\left\{P+\Omega_{i} V^{\alpha-1}\right\}\right)={ }^{P} \mu_{i}^{\infty}+R T \ln \left(\left\{{ }^{s o l} \gamma_{i}\right\}\left\{{ }^{P} C_{i, s a t}\right\}\right)
$$

or 


$$
\left\{P+\Omega_{i} V^{\alpha-1}\right\}=e^{\frac{{ }^{P} \mu_{i}^{\infty}-\mu_{i}^{o}}{R T}} \frac{\left\{{ }^{s o l} \gamma_{i}\right\}}{\left\{{ }^{g} \gamma_{i}\right\}}\left\{{ }^{P} C_{i, s a t}\right\}
$$

We write

$$
{ }^{P} K_{h}=e^{\frac{P}{\mu_{i}^{\infty}-\mu_{i}^{o}}}
$$

${ }^{P} K_{h}$ is the value of the Henry's constant of the gas at the considered temperature. It depends on the pressure $P$. This establishes a general relationship that links the external pressure, the volume of the gaseous aggregates and the concentration at saturation.

$$
\left\{P+\Omega_{i} V^{\alpha-1}\right\}={ }^{P} K_{h} \frac{\left\{{ }^{\text {sol }} \gamma_{i}\right\}}{\left\{{ }^{g} \gamma_{i}\right\}}\left\{{ }^{P} C_{i, s a t}\right\}
$$

Therefore, it must be considered that for a system, at the external pressure $P$, involving a gas phase and a solution, there are an infinite number of equilibrium states for which the concentration of the gas in solution and the phase volume are linked parameters (non-autonomy of the interface). A simplified form of this relationship can be proposed to facilitate the discussion of its implications. The gas solutions are diluted, and it is reasonable to assume that $\left\{{ }^{s o l} \gamma_{i}\right\}=1$.

The internal pressures of confined gases can be high, and obviously their behaviour is no longer that of perfect gases. However, if we refer to the compressibility measurements of usual gases such as nitrogen ${ }^{[9]}$, we can see that there is a clear deviation of $\left\{{ }^{g} \gamma_{i}\right\}$ from the unit only for pressures higher than 200 bars, which corresponds to very small particles of the order of few nanometers in size (applying the Laplace's law). We will assume that we are outside these areas and that $\left\{{ }^{g} \gamma_{i}\right\} \approx 1$.

In addition, since the values of the partial molar volumes of the gases are small ${ }^{[10]}$, it will be approximated that the Henry's constant is independent of the pressure and it only takes into account the nature of the gas and the temperature ${ }^{[11]}$.

So we will discuss the relationship

$$
\left\{P+\Omega_{i} V^{\alpha-1}\right\}=K_{h}\left\{{ }^{P} C_{i, s a t}\right\}
$$

For a given pressure, the smaller the volume of the gaseous aggregate, the higher the concentration in solution $\left(\Omega_{i}>0\right)$.

\subsubsection{Relationships between solubility, size and pressure}

One of the special cases of this relationship corresponds, for a pressure $P$, to the creation for the gas of an "unlimited phase", i.e. having an infinite volume, for the concentration ${ }^{P} C_{i, s a t}^{H}$. These are the conditions under which Henry's law is defined ${ }^{[12]}$, 


$$
\{P\}=K_{h}\left\{{ }^{P} C_{i, s a t}^{H}\right\}
$$

It should be noted that in this particular case, the interface between the solution and the gas does not intervene. The system is considered as the juxtaposition of two autonomous phases (gas and solution). Only the interface is a non-autonomous phase. When the gas is in the form of bubbles, the concentration of solutions is higher than that provided for by Henry's law. The solutions are oversaturated, ${ }^{P} C_{i, s a t}>{ }^{P} C_{i, s a t}^{H}$. To characterize this state, we define oversaturation, $\mathfrak{R}$, such as

$$
\Re=\frac{{ }^{P} C_{i, s a t}}{{ }^{P} C_{i, s a t}^{H}} \geq 1
$$

This magnitude is related to the particle size.

$$
\frac{\left\{P_{N E}\right\}}{\{P\}}=\frac{\left\{P+\Omega_{i} V^{\alpha-1}\right\}}{\{P\}}=\frac{{ }^{P} C_{i, \text { sat }}}{{ }^{P} C_{i, \text { sat }}^{H}}=\mathfrak{R}=1+\left\{\frac{\Omega_{i} V^{\alpha-1}}{P}\right\}
$$

Thus, at a given external pressure $P$, the relationship between the volume of the bubbles, the external pressure and the rate of oversaturation is established as

$$
P V^{1-\alpha}=\frac{\Omega_{i}}{\mathfrak{R}-1}
$$

This relationship shows that Boyle-Mariotte's law ( $P V=$ constant) does not apply to gas entities in solution, simply because gas does not constitute an autonomous phase. The variables $P$ and $V$ are related to each other, but via a third variable which is the concentration of the gas in solution. It is therefore not accurate to build, as in some works, thermodynamic reasoning on this law ${ }^{[13]}$. An illustration of this situation can be given by considering spherical bubbles,

$$
\frac{\left\{P+\frac{2 \gamma}{r}\right\}}{\{P\}}=\frac{{ }^{P} C_{i, s a t}}{{ }^{P} C_{i, s a t}^{H}}=\mathfrak{R}=1+\left\{\frac{2 \gamma}{r P}\right\}
$$

Thus,

$$
r=\left\{\frac{2 \gamma}{P(\mathfrak{R}-1)}\right\}
$$

For an external pressure of $1 \mathrm{bar}$, the values of the radius and volume of the bubble for different values of the oversaturation coefficient are given in Table 1 . The value of the surface tension of the water is taken to be equal to $\gamma \approx 0.072 \mathrm{~N} \mathrm{~m}^{-1}$.

Table 1: variation of the size of spherical bubbles according to the values of the oversaturation coefficient, $\Re$, for an external pressure $P$ of 1 bar.

\begin{tabular}{|ccc|}
\hline$\Re$ & $\mathrm{r} / \mu \mathrm{m}$ & $\mathrm{V} / \mu \mathrm{m}^{3}$ \\
\hline 1 & $\infty$ & $\infty$ \\
1.1 & 14.40 & 12508 \\
1.2 & 7.20 & 1563 \\
\hline
\end{tabular}




\begin{tabular}{|ccc|}
\hline 1.3 & 4.80 & 463 \\
1.4 & 3.60 & 195 \\
1.5 & 2.88 & 100 \\
1.6 & 2.40 & 58 \\
1.7 & 2.06 & 36 \\
1.8 & 1.80 & 24 \\
1.9 & 1.60 & 17 \\
2.0 & 1.44 & 12 \\
2.1 & 1.31 & 9.4 \\
2.2 & 1.20 & 7.2 \\
2.3 & 1.11 & 5.7 \\
2.4 & 1.03 & 4.6 \\
2.5 & 0.96 & 3.7 \\
\hline
\end{tabular}

As can be seen, it is when the oversaturation is lowest that the bubble size becomes the largest. This behaviour is logical since in such conditions, the gas phase becomes unlimited. Experimentally, these situations have been described by different authors. Ohgaki et al. ${ }^{[14]}$ obtained, for $50 \mathrm{~nm}$ nitrogen bubbles in water, an oversaturation $\Re$ of about 36 , German et al. ${ }^{[15]}$ determined a ratio $\Re=310$ for $\mathrm{H}_{2}$, and $\Re=160$ for $\mathrm{N}_{2}$ under the conditions of their electrolysis experiments. In this particular case of nitrogen, Oghaki calculated an internal bubble pressure of 60 bars, whilst the value calculated by Laplace's law is about 30 bars. This remark is important because it raises questions about the validity of the application of the Laplace relationship for these entities, and in particular about the value of the surface tension.

In our opinion, we believe that this behaviour stems from the fact that the solution/gas boundary, in the case of nanobubbles, is not geometrically defined. This boundary is a "fuzzy" interface and thus, the notion of surface tension has no more the usual meaning. Under these conditions, it seems delicate to us to criticize certain works ${ }^{[16]}$, such as those of Azevedo et $a l .{ }^{[17]}$, for the reason that their experimental results do not corroborate those theoretically foreseeable from nucleation theory.

\subsubsection{Effect of the dilution}

When the nanobubble solution is conceived as the juxtaposition of two autonomous phases, its dilution should only result in the dispersion of the bubbles, without any change in size. This is not what Eq.19 provides. By modifying the concentration of the gas in solution, the volume of the bubbles must be changed at constant pressure. For a dilution of an oversaturated solution obtained by decreasing the value of $\Re$, an increase in the volume of the bubbles is expected. This behaviour was recently observed by Tuziuti et al. ${ }^{[18]}$. These authors had diluted an aqueous 
solution of air nanobubbles with degassed water at $23 \pm 1^{\circ} \mathrm{C}$. The decrease in the concentration of dissolved air in the solution has led to an increase in the average size of nanobubbles at constant external pressure. This corresponds to a spontaneous evolution of the solution in order to respect the obligations of the nonextensive thermodynamics expressed by Eq. 15. Tuziuti et al. had also suggested, in the conclusion of their paper, that stable nanobubbles may exist in liquids undersaturated in gas. This hypothesis had previously been put forward by Guo et al. ${ }^{[19]}$. We made the same point in our study on confined gases ${ }^{[20]}$. This can be achieved if $\Omega$ is negative, for the considered medium, which is observed for some coated particle systems.

\subsubsection{Effect of the pressure}

The previous relationships allow discussing the effects of pressure on bubble solutions. Consider, for example, a supersaturated solution at equilibrium whose behaviour responds to Eq. 15. This value of the dissolved gas concentration would correspond to a Henry's equilibrium pressure which would be $P^{H}$, such that

$$
\left\{P+\Omega_{i} V^{\alpha-1}\right\}=K_{h}\left\{{ }^{P} C_{i, s a t}\right\}=\left\{P^{H}\right\}
$$

Consider a sudden increase in external pressure $P$, which leaves the value of the concentration of the solution constant. To maintain the condition $\left\{C_{i, s a t}\right\}=$ constant, the increase in external pressure must be compensated by the associated volume term. This implies that the bubble volume must increase $(\alpha<1)$ ! This evolution can be illustrated by treating the particular case of spherical bubbles.

In this situation, the value of the bubble radius would vary with the pressure according to

$$
\left\{\frac{2 \gamma}{P^{H}-P}\right\}=r
$$

In the latter equation, the surface tension, $\gamma$, is in $\mathrm{N} \mathrm{m}^{-1}$, the pression in $\mathrm{Pa}$ and the radius, $\mathrm{r}$, in $\mathrm{m}$. It can be seen that if the external pressure $P$ increases then the value of $r$ also increases. In Table 2, we have reported the variations in the bubble radius, taking as equilibrium pressure of saturation $P^{H}=3$ bars. We also calculated the bubble volumes and the ratios between the bubble volume to that which corresponds to an initial pressure of 1 bar.

Table 2: Variation of the size of spherical bubbles according to the value of the external pressure, considering a constant oversaturation coefficient, $\mathfrak{R}$ (saturation at $P^{H}=3$ bars) and a surface tension of $72 \mathrm{mN} \mathrm{m}^{-1}$.

\begin{tabular}{|cccc|}
\hline $\mathrm{P} / \mathrm{bar}$ & $\mathrm{r} / \mu \mathrm{m}$ & $\mathrm{V} / \mu \mathrm{m}^{3}$ & ratio \\
\hline 1 & 0.72 & 1.6 & 1.00 \\
1.1 & 0.76 & 1.8 & 1.17 \\
\hline
\end{tabular}




\begin{tabular}{|cccc|}
\hline 1.2 & 0.80 & 2.1 & 1.37 \\
1.3 & 0.85 & 2.5 & 1.63 \\
1.4 & 0.90 & 3.1 & 1.95 \\
1.5 & 0.96 & 3.7 & 2.37 \\
1.6 & 1.03 & 4.6 & 2.92 \\
1.7 & 1.11 & 5.7 & 3.64 \\
1.8 & 1.20 & 7.2 & 4.63 \\
1.9 & 1.31 & 9.4 & 6.01 \\
2 & 1.44 & 12 & 8.00 \\
2.1 & 1.60 & 17 & 10.97 \\
2.2 & 1.80 & 24 & 15.63 \\
2.3 & 2.06 & 36 & 23.32 \\
2.4 & 2.40 & 58 & 37.04 \\
2.5 & 2.88 & 100 & 64.00 \\
2.6 & 3.60 & 195 & 125.00 \\
2.7 & 4.80 & 463 & 296.30 \\
2.8 & 7.20 & 1560 & 1000.00 \\
2.9 & 14.40 & 12510 & 8000.00 \\
3 & $\infty$ & $\infty$ & $\infty$ \\
\hline
\end{tabular}

As shown in Table 2, the bubble volume increases with the pressure, it is much bigger when the pressure gets closer to Henry's equilibrium (here 3 bars). The phenomenon is significant, since the change from 1 to 2 bars leads to an eightfold increase of the bubble volume! To our knowledge, there are few experimental studies that undoubtedly illustrate this behaviour. Tuziuti et al. ${ }^{[20]}$ had studied the influence of increase in static pressure on bulk nanobubbles. The authors had prepared solutions of air nanobubbles, the bubble concentration had been measured using a particle counter before the solution pressurization and after returning to atmospheric pressure. Their measurements concerned particles from 100 to $150 \mathrm{~nm}$ with an increase in pressure of $310^{4} \mathrm{kPa}$, for different times ranging from 1,10 , to $100 \mathrm{~min}$. They showed that the increase in pressure results, for each cycle, in a decrease in the number of bubbles in the solution and an increase in the particle size.

It is difficult to conclude on these manipulations at the thermodynamic level, apart from that at high pressure, the distribution of the gas in solution and in bubbles evolves with as final balance an increase in the bubble size. Especially since in the description of their system they assumed that the bubble size decreases with pressure. This statement is essential to the interpretation they proposed. 


\section{Discussion}

It appears from the previous developments that it is not legitimate using Gibbs' extensive thermodynamics to describe the behaviour of media made of gases "dispersed" in the solution. These results fully corroborate Kaptay's analyses ${ }^{[21]}$. On this subject, we have chosen to illustrate our approach by taking the particular case of entities obeying the Laplace relationship, but we could also have used the notion of specific surface area (3/r), introduced by Kaptay.

We illustrated this situation by discussing a very similar case, that of mixed micelles composed of a mixture of surfactant compounds, for which we showed the "non-applicability" of the Gibbs-Duhem relationship ${ }^{[22]}$.

In the above description, we have given particular importance to the case where, $\tau(\Omega)$, as $\gamma$ was a positive parameter of tension.

However, this is probably not the generality. Kaptay ${ }^{[8]}$ mentioned the possibility that the surface tension could be negative, resulting in a spontaneous emulsification of the solution. German et $a l .{ }^{[15]}$ discussed the modification of surface tension values for smaller bubble sizes. Concerning nonextensive thermodynamics, negative values for the tension term $\tau^{[7]}$ are considered in the case of coated nanoparticles, but also for the micellar phases of charged surfactants ${ }^{[23]}$. In this case, the nonextensive pressure of the bubbles would be lower than the external pressure, and an increase in the latter would result in a decrease in the bubble volume. Thus, the system would evolve as predicted by Boyle Mariotte's relationship, but this would in no way prove their autonomy. Eq. 15 would continue to apply and link the three parameters, pressure, volume and solubility that make the bubble phase non-autonomous. The nonextensive pressure of the bubble would then be lower than the external pressure. On this point, we can mention the work of Johnson et al. ${ }^{[24]}$ on the response to the pressure of air bubbles (of diameter about 40 to 100 $\mu \mathrm{m})$, covered with an organic film, generated in seawater. The increase in pressure leads to a decrease in volume. By the way, another remark seems important to us. We have treated here the case of bubbles with only one solution/gas interface. For nanobubbles put on substrates, or in contact with different walls ${ }^{[25]}$, all interfaces, $k$, that are involved in the equilibrium must be taken into account in the definition relationships of the nonextensive pressure. Then, Eq. 2 becomes

$$
P_{N E}-P=\sum \tau_{k} \frac{d \chi_{k}}{d V}=V^{\alpha-1} \sum \Omega_{k}
$$

This is how we proceeded for wettability ${ }^{[26]}$. The value of $\alpha$ depends on the nature of the considered interface. In this situation, our relationships continue to apply, and there is nothing to prevent from assuming the use of negative tension terms for certain interfaces, leading to an 
internal pressure lower than the external pressure ${ }^{[27]}$ and an increase in volume during a depression $^{[28]}$.

\section{Conclusion}

We mainly show, in this study, that bubble solutions cannot be considered as the simple juxtaposition of autonomous phases of gas and solution. The consequence of this is that the existence of nanobubbles in solution is still discussed ${ }^{[29]}$ and sometimes even contested, very rightly in our opinion, at least in terms of thermodynamics. The existence of gaseous aggregates within supersaturated solutions seems to be no longer in doubt ${ }^{[30]}$, but there is a big difference between saying that and considering them as "objects", there is a gap that should not be crossed, in our opinion. Nanobubble solutions should not be considered as the juxtaposition of autonomous phases, but as particular entities which can be named "supersaturated solutions" where gas is "simultaneously" in two forms (solution and bubble), in permanent exchange. The supersaturated solution is thermodynamically autonomous but its components are not and formally it is not correct to describe them by means of extensive thermodynamics. Boyle Mariotte's law does not apply to gas contained in nanobubbles, which do not form autonomous phases $^{[13]}$. The theoretical relationships proposed in this work constitute another approach to bubble solutions, which takes into account the non-autonomous nature of the constituents supersaturated solutions in gas.

Keywords: Bubbles, Nanobubbles, Thermodynamics, Nonextensive thermodynamics, Solubility, Henry's law 


\section{References}

[1] S. H. Oh, J. G. Han, J.-M. Kim, Fuel 2015, 158, 399-404.

[2] M. Alheshibri, J. Qian, M. Jehannin, V. S. Craig, Langmuir 2016, 32, 11086-11100.

[3] K. Yasui, T. Tuziuti, W. Kanematsu, Ultrason. Sonochem. 2018, 48, 259-266.

[4] P. Letellier, M. Turmine, J. Colloid Interface Sci. 2013, 392, 382-387.

[5] M. Turmine, A. Mayaffre, P. Letellier, J. Phys. Chem. B 2004, 108, 18980-18987.

[6] R. Defay, I. Prigogine, Surface Tension and Adsorption, Longmans, Green \& Co Ltd, London, 1966.

[7] P. Letellier, A. Mayaffre, M. Turmine, Phys. Rev. B 2007, 76, 045428_1-9.

[8] G. Kaptay, Langmuir 2017, 33, 10550-10560.

[9] A. Michels, H. Wouters, J. De Boer, Physica 1936, 3, 585-589.

[10] T. L. Zhou, R. Battino, J. Chem. Eng. Data 2001, 46, 331-332.

[11] W. A. Gerth, Arch. Biochem. Biophys. 1985, 241, 187-199.

[12] R. Sander, Atmos. Chem. Phys. 2015, 15, 4399-4981.

[13] R. P. Berkelaar, E. Dietrich, G. A. Kip, E. S. Kooij, H. J. Zandvliet, D. Lohse, Soft Matter 2014, 10, 4947-4955.

[14] K. Ohgaki, N. Q. Khanh, Y. Joden, A. Tsuji, T. Nakagawa, Chem. Eng. Sci. 2010, 65, 1296-1300.

[15] S. R. German, Q. Chen, M. A. Edwards, H. S. White, J. Electrochem. Soc. 2016, 163, H3160H3166.

[16] Z. A. Zhou, Miner. Eng. 2018, 117, 117-120.

[17] A. Azevedo, R. Etchepare, S. Calgaroto, J. Rubio, Miner. Eng. 2016, 94, 29-37.

[18] T. Tuziuti, K. Yasui, W. Kanematsu, Ultrason. Sonochem. 2018, 43, 272-274.

[19] Z. Guo, Y. Liu, Q. Xiao, X. Zhang, Langmuir 2016, 32, 11328-11334.

[20] T. Tuziuti, K. Yasui, W. Kanematsu, Ultrason. Sonochem. 2017, 38, 347-350.

[21] a)G. Kaptay, RSC Adv. 2017, 7, 41241-41253; b)G. Kaptay, Adv. Colloid Interface Sci. 2018, 256, 163-192.

[22] P. Letellier, M. Turmine, J. Phys. Chem. B 2015, 119, 4143-4154.

[23] P. Letellier, A. Mayaffre, M. Turmine, J. Colloid Interface Sci. 2008, 321, 195-204.

[24] B. D. Johnson, R. C. Cooke, Science 1981, 213, 209-211.

[25] Y. Sun, G. Xie, Y. Peng, W. Xia, J. Sha, Colloids Surf. A 2016, 495, 176-186.

[26] P. Letellier, A. Mayaffre, M. Turmine, J. Colloid Interface Sci. 2007, 314, 604-614.

[27] M. A. Chappell, S. J. Payne, Respir. Physiol. Neurobiol. 2006, 152, 100-114.

[28] J. Li, H. Chen, W. Zhou, B. Wu, S. D. Stoyanov, E. G. Pelan, Langmuir 2014, 30, 4223-4228.

[29] N. Nirmalkar, A. W. Pacek, M. Barigou, Langmuir 2018, 34, 10964-10973.

[30] F. Y. Ushikubo, T. Furukawa, R. Nakagawa, M. Enari, Y. Makino, Y. Kawagoe, T. Shiina, S. Oshita, Colloids Surf. A 2010, 361, 31-37. 


\section{solvent}

Fuzzy interface

i, solution

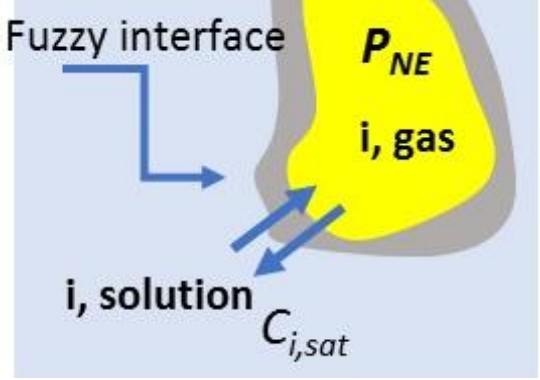

P

Generalized Henry's Law for (nano)bubbles with ill-defined geometrical boundary

$$
P_{N E}=P+\Omega_{i} V^{\alpha-1}=K_{h}{ }^{P} C_{i, s a t}
$$

